\title{
ARTICLE \\ Design and Realization of Automatic Warehouse Based on S7-1500PLC
}

\section{Fuhua Yang* Haitao Li}

Huizhou Technician Institute, Department of electronic engineering, Huizhou 516003, China

\begin{tabular}{l}
\hline ARTICLE INFO \\
\hline Article history \\
Receiced: 24 February 2021 \\
Accepted: 12 April 2021 \\
Published Online: 20 April 2021 \\
\hline Keywords: \\
Automatic warehouse \\
S7-1500 PLC \\
RFID sensor \\
GRAFH language
\end{tabular}

\section{Introduction}

With the rapid development of the logistics industry and the popularization of automated production lines, the production cycle of products has been greatly shortened. Traditional warehouses can no longer meet the requirements. Intelligent warehousing systems can effectively coordinate the production process to achieve accurate and efficient handling and storage of items, which is smart manufacturing. ${ }^{[1]}$

RFID is an automatic identification system, which can identify the target and collect data through the non-contact $\mathrm{RF}$ signal of the electronic tag. It can support fast reading and writing, non visual identification, mobile identification, multi-target identification, and has the function of encrypted communication. RFID has great advantages because of its high data transmission rate and long-distance reading. It can adapt to the harsh industrial environment

\begin{abstract}
In order to improve the efficiency of automatic warehouse control system, the experimental platform of stereoscopic warehouse with s7-1500plc is designed. The manipulator is driven by stepper motor and servo motor to realize $\mathrm{x}, \mathrm{y}$ and $\mathrm{Z}$ three-axis space motion. The material transmission system is built by general-purpose G120 inverter. HMI KTP700 realizes control and status monitoring. The materials are identified and classified by RFID sensor and other sensors.TIAV15 software build PROFINET communication and PROFIBUS communication network.Using the GRAPH language programming can improve the visualization degree of application and solve the complex problems of program design and debugging of the warehouse control system. Through the design of hardware and software, a set of complete control system design scheme is formed, which has high practical value and provides an excellent teaching and experiment platform for the intelligent storage system.
\end{abstract}

and is widely used in logistics, production, retail, transportation and other industries. It has gradually become an indispensable technical means for enterprises to improve the level of material supply chain management, reduce costs and realize enterprise management informatization ${ }^{[2]}$.

In this paper, based on s7-1500 PLC, according to the characteristics of automated warehouse, the system hardware and software design is completed. In the hardware design, the reasonable selection of PLC, inverter and so on, draws the corresponding hardware connection diagram and wiring diagram. In the software design, a complete set of stereoscopic warehouse control system is designed, and the program is designed to improve the storage efficiency of the warehouse.

\section{Hardware Design of the System}

The automatic warehouse system is composed of

*Corresponding Author:

Fuhua Yang,

Huizhou Technician Institute, Department of electronic engineering, Huizhou 516003, China;

Email: 345023575@qq.com 
control management unit, material transmission unit and classified storage unit. The system structure is shown in Figure 1.

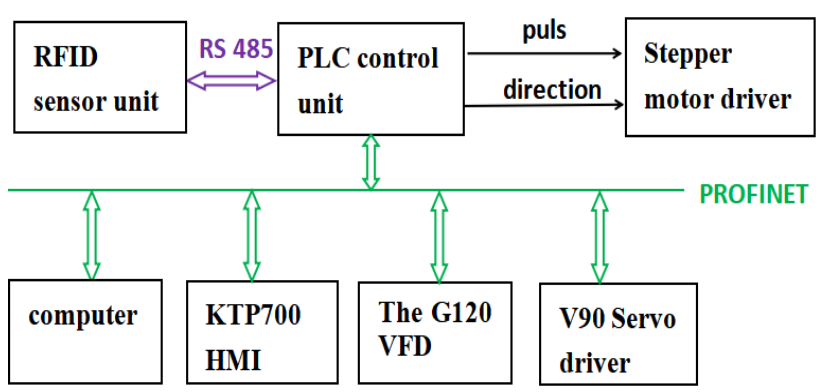

Figure 1. system composition diagram

\subsection{Control Management Unit}

The control system is controlled by PLC, because PLC has good anti-interference ability, stability and communication ability. The programming system is simple and intuitive, which provides a guarantee for the realization of three-dimensional warehouse. The system selects Siemens 1500 series PLC, the model is 1516-3PN / DP, the advantages of this model are: PLC integration has three PN interfaces, a DP interface, can be directly inserted into the communication cable to achieve PROFINET and PROFIBUS communication, do not need to build external communication module.By carrying the process module TM PTO4 with four-channel high-speed pulses, the stepper motor can be controlled; the GRAPH language inside the 1500 series PLC makes the programming of process control more intuitive and convenient for monitoring and debugging. Profinet communication can realize real-time data interaction and interconnection with the Internet. The system network is simple, compatible and extensible. It can use shielded industrial Ethernet cable improves the stability of the system. The communication between S71500 and the RFID sensor adopts the RS485-based PROFIBUS DP protocol, which has good system stability and strong expansion capability. It can read and write electronic tags through the industrial identification instructions in Siemens S7-1500.

The man-machine interface selects KTP700 Basic, 7 -inch touch screen, $1 \times$ PROFINET interface, $1 \times$ USB interface, to meet the control and monitoring requirements of the system. The operator sends commands through the touch screen and sends it to the PLC. The PLC reads the position information of the servo and stepper motors through the sensor signal and sends it to the touch screen. It can display the current position and movement status of the handling manipulator, and read the color attributes of the current material, etc.

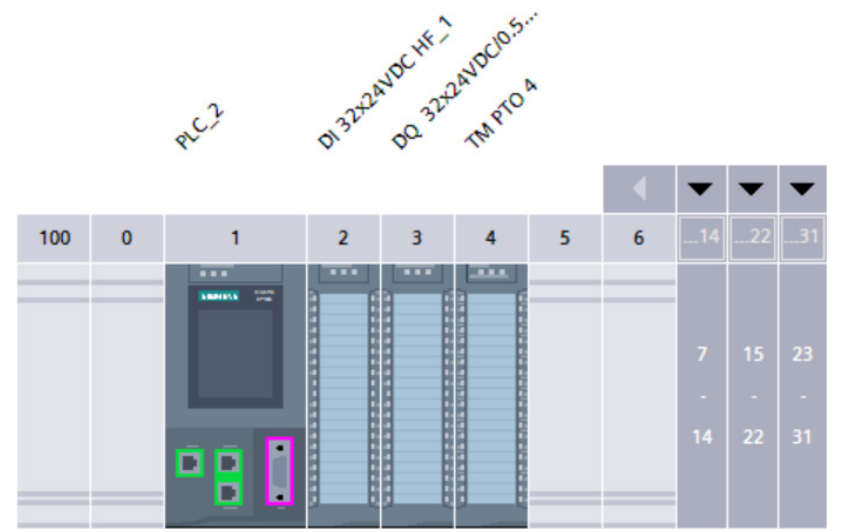

Figure 2. 1516-3pn / DP series PLC

\subsection{Material Transfer Unit}

The material conveying unit uses a squirrel-cage threephase asynchronous motor to drive the conveyor belt, and the Siemens G120 series inverter is used to control the high and low speed operation of the material conveying motor, which can realize accurate parking, and has high safety during operation, and can achieve long distances. Distance control, with strong communication function. The three-phase asynchronous motor of the conveyor belt is directly connected to the G120 inverter, where R. S. $\mathrm{T}$ are connected to the three-phase power supply, PE is grounded, U. V. and $\mathrm{W}$ are connected to the three-phase input of the motor, and the inverter is connected to the CPU of the PLC through PROFINET. The CPU controls the speed of the conveyor motor by sending the control word of the inverter, and reads the speed of the motor by receiving the status word.

\subsection{Handling and Storage Unit}

\subsubsection{Composition of Handling Storage Unit}

The handling and storage unit is composed of three-dimensional shelves and three degree of freedom manipulator, which realizes the handling and classification of materials. The shelves are designed as two $90^{\circ}$ three-layer shelves. Each layer stores the same material and the same color of materials, realizing the storage of materials in and out of the warehouse.The manipulator can realize the spatial movement of $X, y$ and $Z$ axes, in which the movement of $\mathrm{X}$ and $\mathrm{Y}$ axes is controlled by stepper motor, and the movement of $\mathrm{Z}$ axis is controlled by servo motor. The three axes drive the worktable to do three-dimensional movement on the guide rail by using the lead screw, and the clamping part is pneumatic manipulator, so as to realize the storage and delivery of products. A color sensor and an RFID sensor are installed on the upper end of the 
manipulator to identify the material information. RFID system consists of PROFIBUS communication module asm456, electronic tag, reader and antenna. ${ }^{[3]}$ In order to improve the reliability of RFID reading information in metal shelves, the most commonly used method is to paste a layer of magnetic absorbing material behind the RFID tag. The high-frequency anti-metal tag is selected, which is an electronic tag packaged with anti-magnetic absorbing material.The warehouse design is based on the storage and delivery of the production process, and realizes the transportation of materials from shelf 1 to shelf 2 . The middle part is the transportation mechanism, and the status of shelf 1 and shelf 2 can be observed on the outside of the warehouse. The 3D concept photo and real photo are shown in Figure 3.

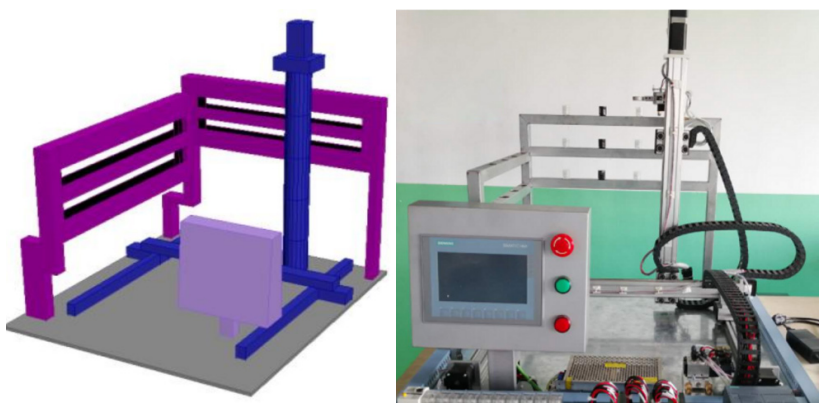

Figure 3. The 3D concept photos and real photos

\subsubsection{Handling Mechanism Wiring}

The 1500 series PLC does not have its own highspeed pulse port, it needs to configure the pulse / direction interface process module tmpto4. With this process module, at most four stepper motor shafts can be connected for a s7-1500 system. The module is connected to the process object through profidrive frame 3 , and forms the driver interface. The pulse / direction interface consists of two signals, in which the frequency of pulse (P) output represents the speed and the number of pulses output represents the travel distance. The direction (d) output is used to define the direction of travel. The wiring diagram of process module and step driver is shown in Figure 4 and Figure 5.

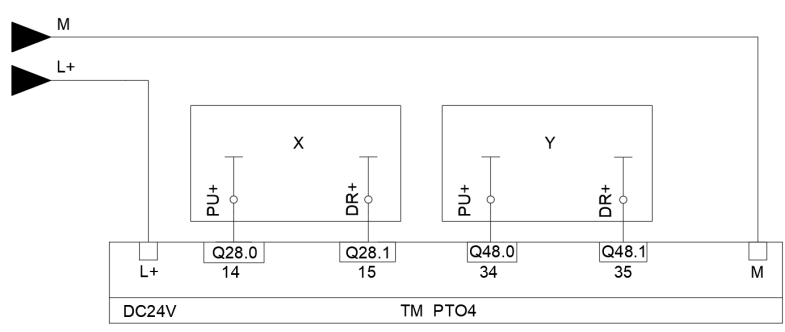

Figure 4. Module TM pto4 wiring diagram

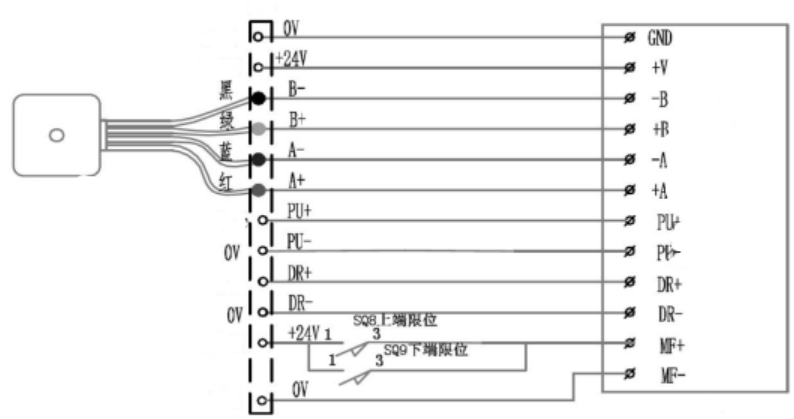

Figure 5. wiring diagram of stepping driver

The servo driver V90 communicates with s7-1500 through profinet.

SINAMICS-V90-PN is configured in TIA V15 software to configure the address range of message and input address I and Q. The SINAMICS-V90 wiring is shown in Figure 6.

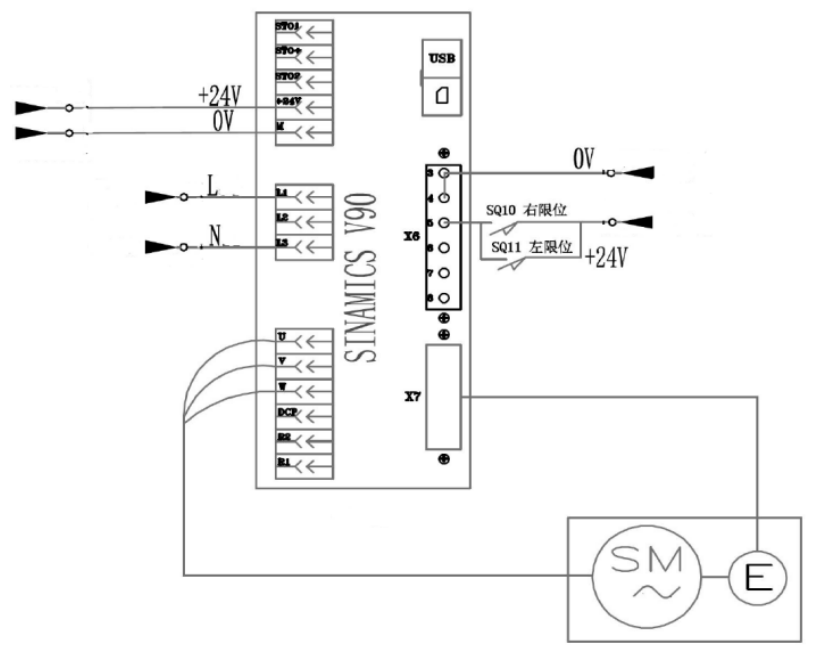

Figure 6. Wiring diagram of SINAMICS-V90 servo drive

\section{Design of Control System of Stereoscopic Warehouse}

According to the functions of automated warehouse, there are mainly the following aspects: communication function; action control: horizontal and vertical movement. Position control: positioning in the reclaiming position, storage position, etc. ; Speed control ;human machine interface.

\subsection{PLC Control System Configuration}

The control system consists of profinet communication and RS485 bus protocol communication. S7-1500plc communicates with inverter, servo driver and touch screen through profinet. 485 communication protocol is used 
with RFID sensor. It is linked with RFID reader through profibus communication module asm456. Through TIA software v15.1, the configuration diagram is shown in Figure 7.

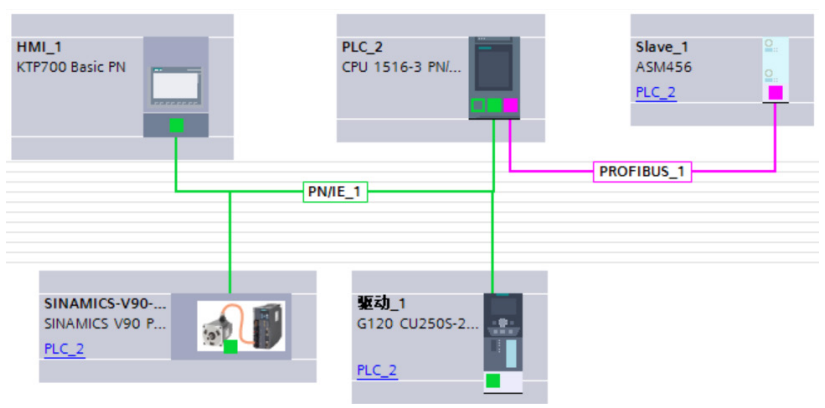

Figure 7. the configuration diagram

\subsection{PLC Program Design}

\subsubsection{Program Flow Chart}

The work flow chart of stereoscopic warehouse system is shown in the Figure 8. Using graph language in s7-1500 to write the sequence control program according to the flow chart can directly reflect the working process of the system. The graphical programming interface facilitates the debugging of the program and improves the programming efficiency and accuracy ${ }^{[4]}$.

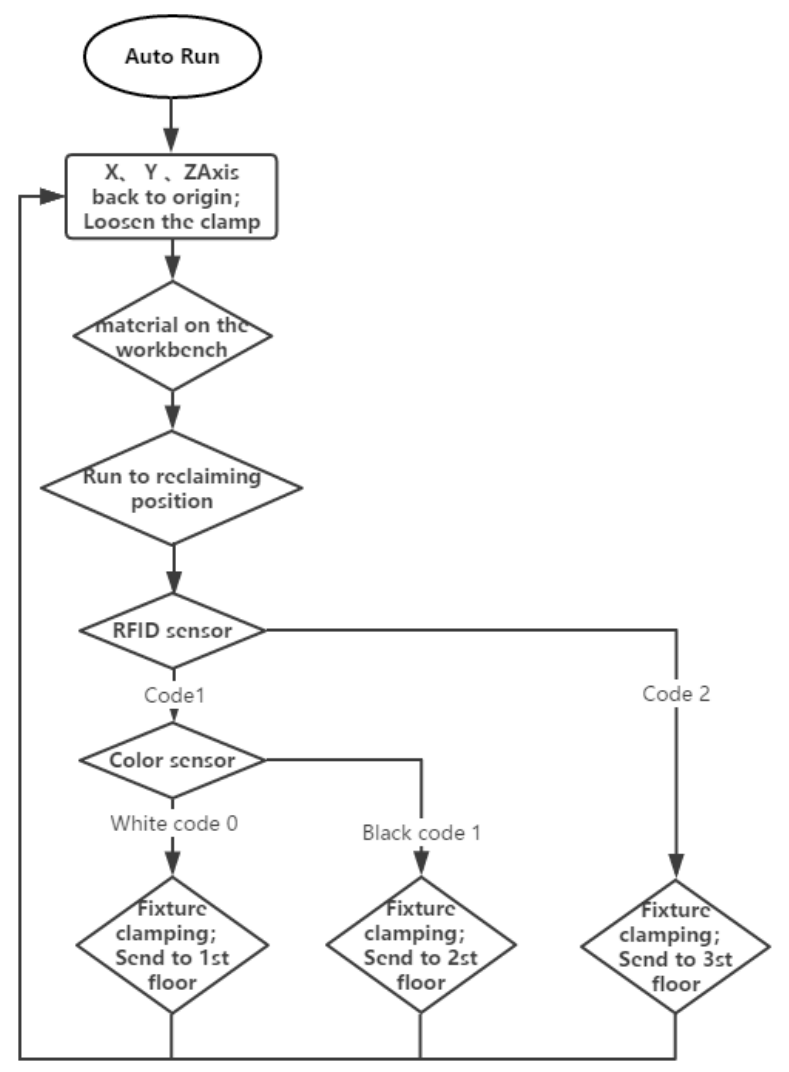

Figure 8. Auto mode program flow chart

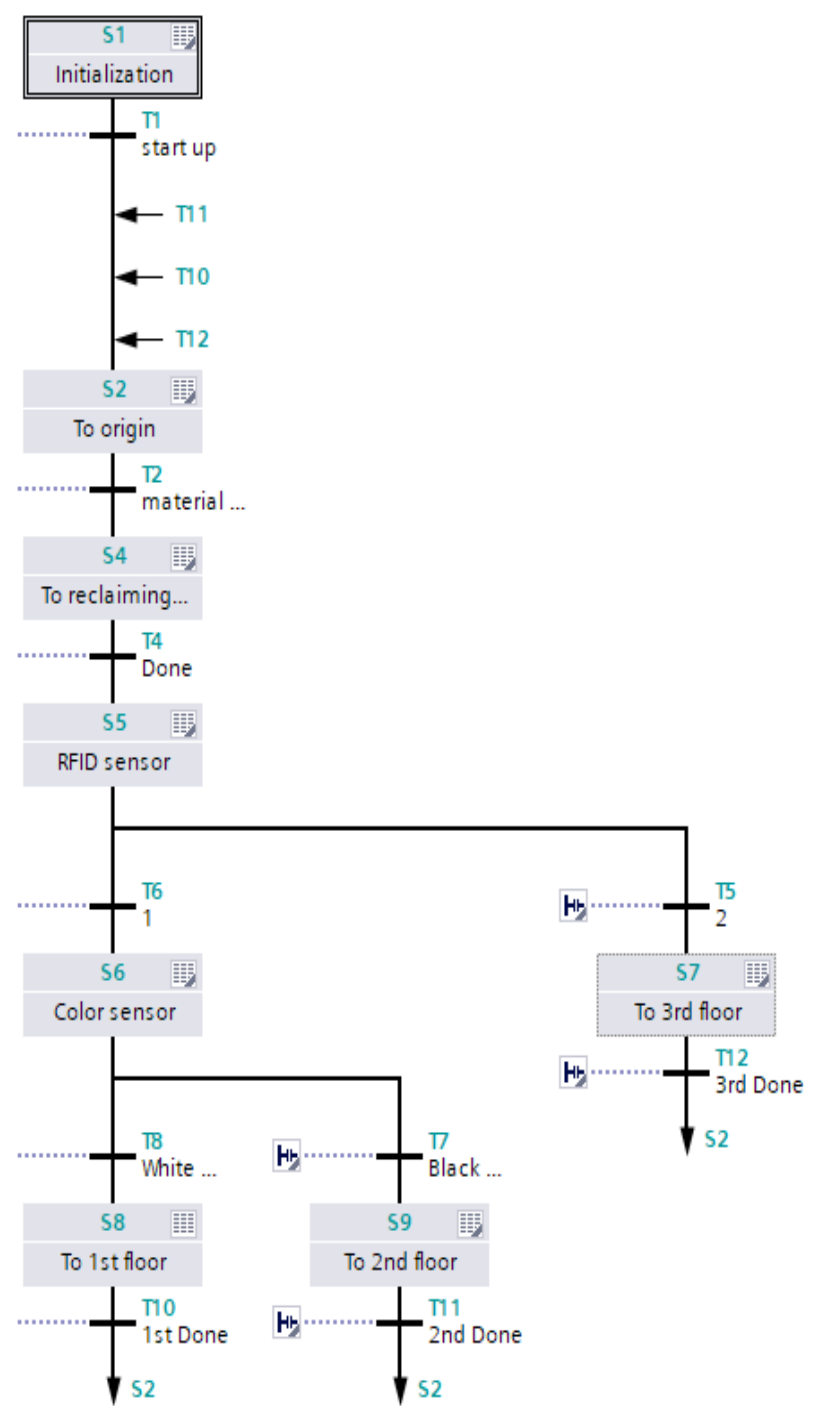

Figure 9. Auto mode GRAPH program

\subsubsection{Program Design}

Take sending materials to the first floor of the warehouse as an example to analyze the program. Create a new FC block named sent to the first floor, and program in the block. After writing, you can drag FC blocks into step S8, S9, S7. The FC block can be called twice or three times to reduce the workload of programming. This paper introduces the functions of some programs in FC block, using the motion control instruction "MC"_ The axis can be moved to an absolute position, the position relative to the origin is defined by the parameter "position", and the dynamic behavior of motion control is defined by "velocity", "jerk", "acceleration" and "deceleration". The X axis determines position control program is shown in the Figure 10 . 


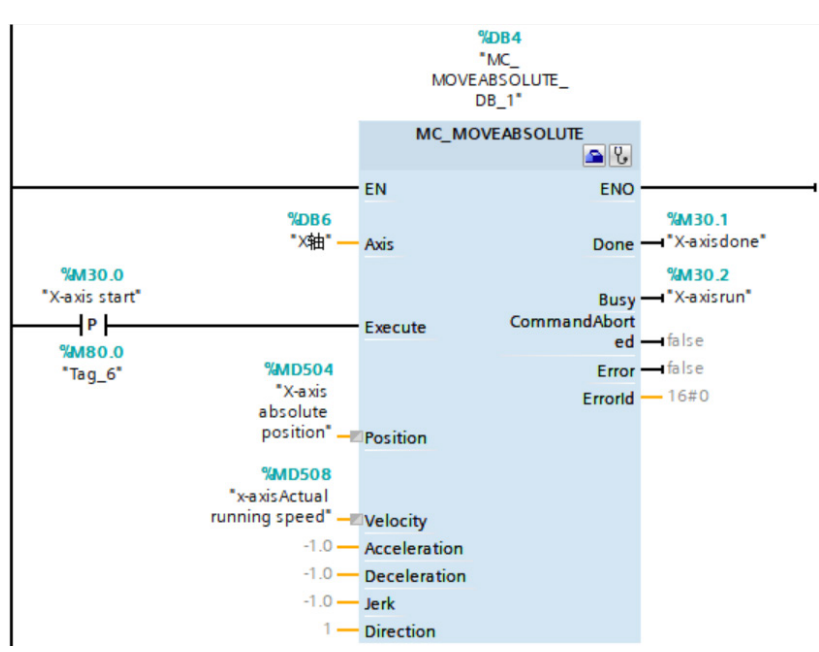

Figure 10. $\mathrm{X}$ axis determines position control program

Because the space between rows and columns of the three-dimensional shelf is fixed, after determining the spatial coordinates of the first row, the subsequent position can be determined by using the automatic address recognition algorithm to determine the coordinates of other rows and columns. Take the first action of putting materials into the stereoscopic warehouse as an example. The z-axis coordinate and $y$-axis coordinate have been fixed. Only the $\mathrm{x}$-axis coordinate changes according to the different bin level. The $\mathrm{x}$-axis coordinate of each bin level is:

$$
\mathrm{L}_{\mathrm{x}}=\mathrm{X}_{1}+\left(\mathrm{N}_{\mathrm{x}}-1\right) \mathrm{L}_{1}
$$

$\mathrm{X}_{1}$ represents the $\mathrm{x}$-axis coordinates of the first bin in the first row, $\mathrm{N}_{\mathrm{x}}$ represents the bin number, and $\mathrm{L}_{1}$ represents the $\mathrm{x}$-axis spacing of each bin. The positioning method of Z-axis and y-axis is the same.

In order to improve the operation efficiency of three-dimensional warehouse, due to the different positions of different bin material level from the reclaiming point, if the fixed speed is used, the storage and delivery time of the distant bin material level is longer, which leads to the low efficiency of storage. Through experiments, the storage and delivery time of each bin is set as $5 \mathrm{~S}$, the maximum speed Vmax and the minimum speed Vmin are set, and the absolute position coordinate is divided by the operation time If the speed is within the effective range, it means that the speed value is effective ${ }^{[5]}$. The $\mathrm{X}$ axis speed setting program is shown in the Figure 11.

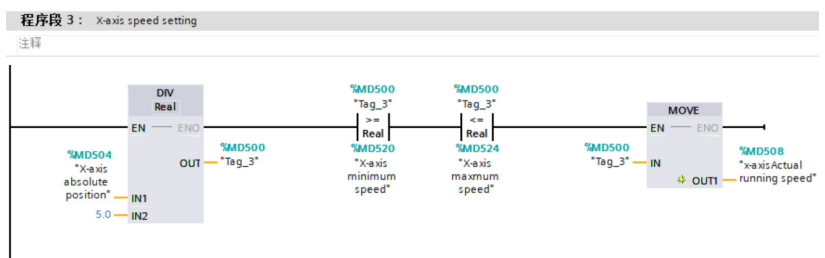

Figure 11. The $\mathrm{X}$ axis speed setting program

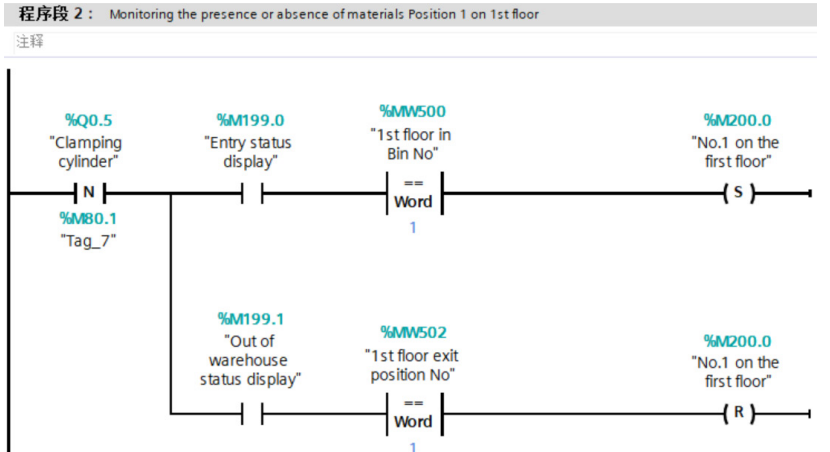

Figure 12. Monitoring the presence or absence of materials on Position 1on $1^{\text {st }}$ floor

\subsection{HMI Design}

According to the system control requirements, the automatic and manual control interface is developed. The screen can realize the system start, stop and other functions. At the same time, it can monitor the real-time position and speed of the manipulator, and display the material number, color and storage status. The manual operation interface is shown in the Figure 13, and the automatic operation interface is shown in the Figure 14.

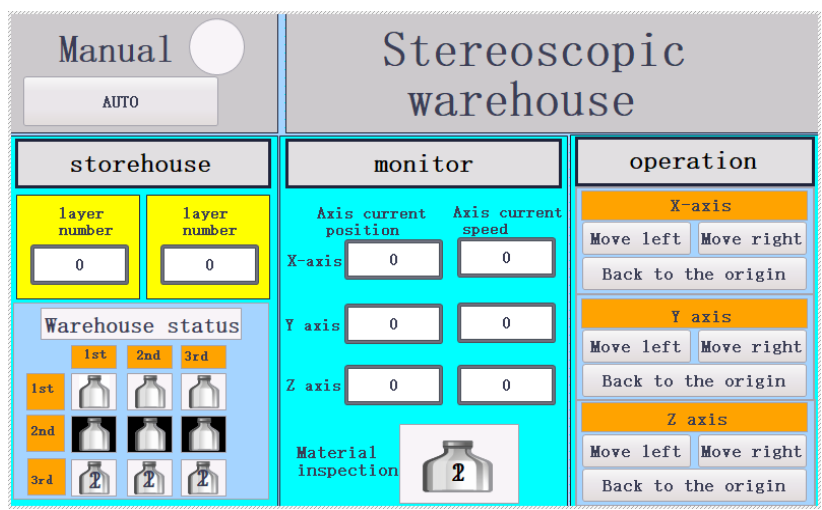

Figure 13. manual operation interface

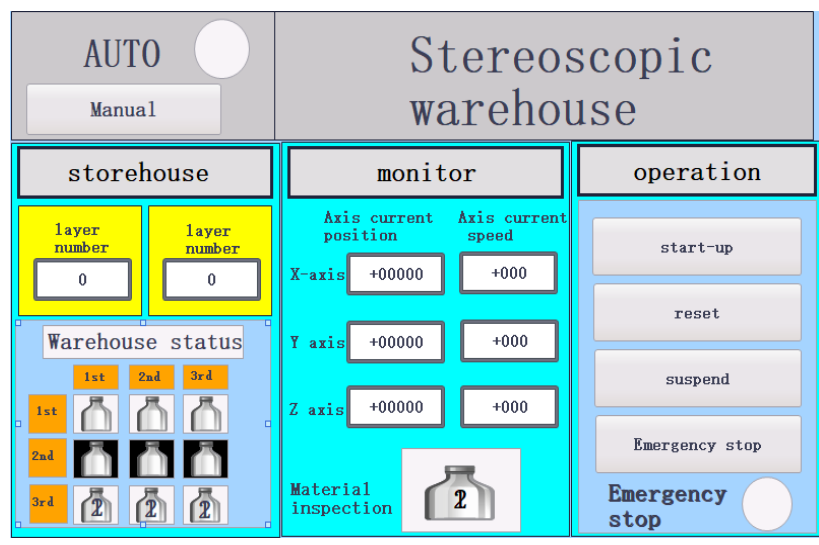

Figure 14. automatic operation interface

Not only need to pay attention to the safety of PLC and 
host computer software, the safety of HMI should also be valued.Once the attacker has successfully compromised the HMI device, he can read or even maliciously modify the data variables in the industrial field.Use a password to restrict access to the HMI local setup menu.Uploading or downloading new projects to the HMI can be protected by a password, and the data stored in the HMI can be retrieved. Access from connected devices (eg PC, PLC) is also password protected.KTP700HMI has two Ethernet ports. These ports provide physical isolation between the local network and the Web.

\section{Conclusions}

The test of the three-dimensional warehouse can meet the design requirements. Based on S7-1500, this article uses frequency conversion technology and stepping servo drive control to realize the three-dimensional warehouse control system. The hardware selection, network construction and program design of the automated three-dimensional warehouse have been completed, and materials can be sorted and stored according to their numbers and colors. The operation mode is efficient, and the control accuracy is high, which is convenient for maintenance and expansion.

\section{References}

[1] ShangYR, WangZ, ZhangKY:Design of Experimental Model of Stereoscopic Warehouse Based on PLC. Journal of Automation Technology and Application. Vol 39 No.10, 55-60,2020.

[2] LuR:Application of RFIDTechnology Automated Storage System.Journal of Automation Technology. Vol 07No.020,57-58,2020.

[3] FengSX:Serial communication between Siemens PLC and RFID reader.Journal of mechanical engineering and automation.2,198-200,(2020.4).

[4] YanL,WangM,LiRP:Design of control system for small stereoscopic warehouse based on S7-1200 PLC.Journal of manufacturing automation.11,10-22. (2020.11).

[5] LiaoCC.S7-1200 programming and application, 3rd Edition.Beijing:China Machine Press.(2017). 